OPEN ACCES

\title{
Anionic Surfactant Based Topical Curcumin Nanosuspension: Fabrication, Characterization and Evaluation
}

Moorthi Chidambaram $^{1 *}$, Senthilkumar Chinnamaruthu², Mohan Sellappan ${ }^{2}$, Kathiresan Krishnasamy ${ }^{1}$

${ }^{1}$ Department of Pharmacy, Annamalai University, Annamalai Nagar, Chidambaram, Tamil Nadu, India

${ }^{2}$ Department of Pharmacy, Shri JJT University, Vidyanagari, District-Jhunjhunu, Rajasthan, India

* Corresponding author: cmoorthitgodu@gmail.com

\begin{abstract}
Curcumin, a hydrophobic polyphenol isolated from dried rhizomes of turmeric exhibits diverse pharmacological activities including anti-bacterial. However, the clinical usefulness of curcumin was limited mainly due to low aqueous solubility and stability. The primary aim of the study was to prepare anionic surfactant based curcumin nanosuspension and to assess its in-vitro anti-bacterial efficacy on Escherichia coli in comparison with ethanolic solution of curcumin. Curcumin nanosuspension was prepared by nanoprecipitation method, characterized for the average particle size, span, uniformity, surface area, and surface morphology and assessed for its anti-bacterial activity against gram-negative bacteria Escherichia coli using agar well diffusion method. Prepared curcumin nanosuspension showed an average particle size of about $175 \mathrm{~nm}$, span of about 1.5 , uniformity of about 0.8 , surface area of about $57 \mathrm{~m}^{2} / \mathrm{g}$ and nanoparticles were spherical in shape. Curcumin nanosuspension have shown significant $(\mathrm{P}<0.05)$ anti-bacterial activity against Escherichia coli at various concentrations in comparison with ethanolic solution of curcumin. We conclude that the size reduction of curcumin in nano range has increased the surface area resulting in increased aqueous solubility and reactability of curcumin, which in turn have increased the potency of curcumin nanosuspension.
\end{abstract}

Keywords: Anionic Surfactant, Curcumin, Nanoprecipitation Method, Nanosuspension, Sodium Lauryl Sulfate

Citation: M. Chidambaram et al. Anionic Surfactant Based Topical Curcumin Nanosuspension: Fabrication, Characterization and Evaluation. Nano Biomed. Eng. 2013, 5(2), 86-89.

DOI: $10.5101 /$ nbe.v5i2.p86-89.

\section{Introduction}

Curcumin (diferuloylmethane), a hydrophobic polyphenol isolated from dried rhizomes of turmeric (Curcuma longa Linn.) exhibits diverse pharmacological activities including anti-inflammatory, anti-oxidant, and anti-bacterial due to wide spectrum of molecular targets and useful in the treatment of various diseases including acquired immune deficiency syndrome, allergy, alzheimer's disease, arthritis, atherosclerosis, cancer, cataract, cystic fibrosis, diabetes mellitus, epilepsy, fever, gall stones, gastric ulcer, hypothyroidism, inflammatory bowel disease, leishmaniasis, lung diseases, malaria, multiple sclerosis, myocardial infarction, osteoporosis, pancreatitis, parkinson's disease, psoriasis, scleroderma, and wound [1-5].

However, the clinical usefulness of curcumin was limited mainly due to low aqueous solubility and stability. Many approaches have been tried to enhance curcumin aqueous solubility, which includes albumin nanoparticles, co-solvent, cyclodextrin inclusion, liposome, magnetic nanoparticles, microcapsule, microsphere, nanosponges, phospholipids complexes, polymer micelles, polymeric nanoparticles, solid dispersion, solid lipid nanoparticles and surfactant free nanoparticles [6,7]. However, anti- bacterial activity of anionic surfactant based curcumin nanosuspension was not yet reported. Hence, the primary aim of the study was to prepare anionic surfactant based curcumin nanosuspension and to assess its in-vitro antibacterial efficacy on Escherichia coli in comparison with ethanolic solution of curcumin.

\section{Experimental section}

\subsection{Materials}

The chemicals used in this study were obtained from commercial sources and used as received without any further purification. Curcumin, $\beta$-cyclodextrin, nutrient broth and nutrient agar were obtained from Himedia Laboratories (Mumbai, India). Analytical grade ethanol was obtained from Brampton (Ontario, Canada). Sodium Lauryl Sulfate was obtained from S.D Fine Chemicals (Mumbai, India).

\subsection{Fabrication of anionic surfactant based topical curcumin nanosuspension}

Anionic surfactant based topical curcumin nanosuspension was prepared based on nanoprecipitation method 
$[2,6]$. Briefly, $100 \mathrm{mg}$ of curcumin was dissolved in 20 $\mathrm{ml}$ of ethanol and sonicated (Lark, India) for 5 minutes. The prepared organic phase was then added to $50 \mathrm{ml}$ of aqueous phase containing $50 \mathrm{mg}$ of sodium lauryl sulphate and $50 \mathrm{mg}$ of $\beta$-cyclodextrin under the influence of sonication $(40 \mathrm{kHz}$; Lark, India) for 60 minutes. Prepared curcumin nanosuspension was subjected to ultra centrifugation (Remi, India) at 19,000 rpm for about 45 minutes at $-20^{\circ} \mathrm{C}$ to separate sodium lauryl sulphate coated curcumin nanoparticles, which was washed and re-suspended in distilled water and used for further characterization.

\subsection{Characterization of anionic surfactant based topical curcumin nanosuspension}

The average particle size, span, uniformity and surface area of the prepared anionic surfactant based topical curcumin nanosuspension was measured based on laser light scattering principle using Mastersizer (Malvern, UK) [6]. Briefly, prepared curcumin nanosuspension was added drop-wise in to the water maintained in the sample dispersion unit of particle size analyser, where the nanoparticles scattered using single shaft pump and stirrer and re-circulated continuously around the measurement zone of the particle size analyser. The surface morphology of the prepared curcumin nanosuspension was determined by transmission electron microscopy (Hitachi H7500) at 20,000 magnifications.

\subsection{In-vitro anti-bacterial assay of anionic surfac- tant based topical curcumin nanosuspension}

Gram-negative bacteria Escherichia coli were used to study the anti-bacterial activity of anionic surfactant based topical curcumin nanosuspension using agar well diffusion method [8]. Briefly, sterilized nutrient agar media was poured into standard petri plates $(60 \times 15$ $\mathrm{mm}$ ) with $4 \mathrm{~mm}$ depth and allowed to solidify at room temperature. Escherichia coli were then inoculated on the solidified agar plate. A $3 \mathrm{~mm}$ wide well/cavity was made using a sterilized steel borer in each petri plates. Various concentrations of prepared anionic surfactant based topical curcumin nanosuspension and ethanolic solution of curcumin were added to corresponding well/cavity using micropipette. The petri plates were maintained undisturbed at room temperature for 30 minutes to allow the diffusion of samples and then incubated for 24 hours at $37^{\circ} \mathrm{C}$. Zone of inhibition diameter was measured after incubation period. The entire assay was carried out under aseptic condition in triplicate. The anti-bacterial potential of the samples were assessed based on the mean diameter of zone of inhibition around the wells.

\subsection{Statistical analysis}

The in-vitro study results were expressed as mean \pm standard deviation (SD) and student's t-test was used to assess the difference between groups using GraphPad Prism software (version 5.04). The differences were considered significant if $\mathrm{P}$ value $<0.05$ and nonsignificant if $\mathrm{P}$ value $>0.05$.

\section{Results and Discussion}

\subsection{Fabrication of anionic surfactant based topical curcumin nanosuspension}

Anionic surfactant based topical curcumin nanosuspension was prepared based on nanoprecipitation method. During fabrication, addition of organic phase in to aqueous phase leads to rapid miscibility of ethanol with water resulting in initiation of curcumin nucleation. Simultaneously, sonication process produces cavitation (bubbles), which oscillates nonlinearly and ultimately collapse resulting in production of high temperature, high pressure, and shocking wave, which inhibits curcumin nucleation at the initial stage. However, nucleated curcumin forms complex with $\beta$-cyclodextrin and increases the solubility of curcumin in the aqueous phase. Subsequently, anionic surfactant sodium lauryl sulfate get adsorbed on the formed complex. Anionic nature of the surfactant provides higher zeta potential to the formed nanoparticles and develops an electrostatic force, which maintains the nanoparticles in Brownian motion and overcomes the Van der Waals force of attraction

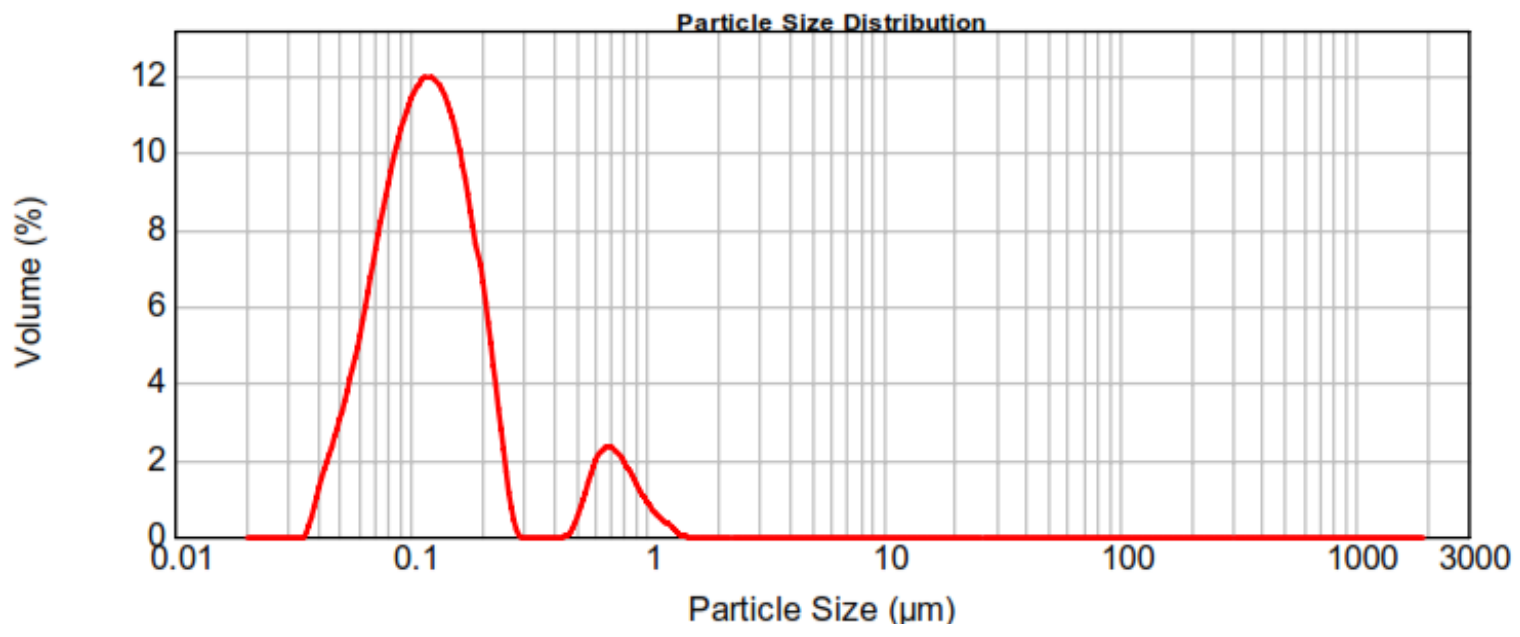

Fig. 1 Particle size distribution of prepared anionic surfactant based topical curcumin nanosuspension . 
and gravitational force resulting in the prevention of nanoparticle aggregation and sedimentation. Though sodium lauryl sulphate coated curcumin nanoparticles were formed instantaneously, sonication process was continued up to 60 minutes to remove the residual ethanol present in the curcumin nanosuspension. Prepared curcumin nanosuspension was subjected to ultra centrifugation at 19,000 rpm for about 45 minutes at $-20^{\circ} \mathrm{C}$ to separate sodium lauryl sulphate coated curcumin nanoparticles and washed with distilled water to remove the free curcumin, sodium lauryl sulphate, and $\beta$-cyclodextrin.

\subsection{Characterization of anionic surfactant based topical curcumin nanosuspension}

Prepared curcumin nanosuspension was characterized for average particle size, surface area, span, uniformity and morphology as these parameters determines the solubility, cellular uptake and consistency of performance. Prepared curcumin nanosuspension showed an average particle size of about $175 \mathrm{~nm}$, span of about 1.5, uniformity of about 0.8 and surface area of about $57 \mathrm{~m}^{2} / \mathrm{g}$ (Fig. 1). Transmission electron microscopy (TEM) image of prepared curcumin nanosuspension has shown spherical morphology of sodium lauryl sulphate coated curcumin nanoparticles at 20,000 magnifications (Fig. 2).

\subsection{In-vitro anti-bacterial assay of anionic surfac- tant based topical curcumin nanosuspension}

Prepared anionic surfactant based topical curcumin nanosuspension was studied for its anti-bacterial activity against gram-negative bacteria Escherichia coli at various concentrations in comparison with ethanolic solution of curcumin and the results are summarized in table 1 . At $50 \mu \mathrm{L}$ concentration, ethanolic solution of curcumin has produced about $14 \mathrm{~mm}$ zone of inhibition and further increase in concentration to $100 \mu \mathrm{L}$ and 150 $\mu \mathrm{L}$ has produced only $16 \mathrm{~mm}$ zone of inhibition, which shows the non-linearity in response when increase in concentration. However, $50 \mu \mathrm{L}$ concentration of curcumin nanosuspension has produced $21 \mathrm{~mm}$ zone of inhibition

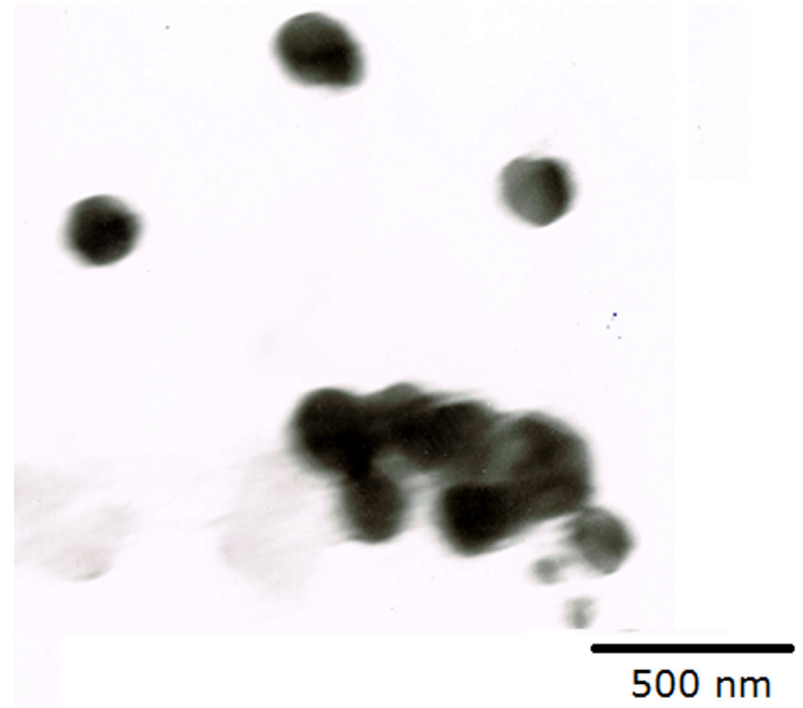

Fig. 2 TEM image of anionic surfactant based topical curcumin nanosuspension.

and further increase in concentration to $100 \mu \mathrm{L}$ and 150 $\mu \mathrm{L}$ has produced $25 \mathrm{~mm}$ and $29 \mathrm{~mm}$ zone of inhibition, which shows the linearity in response when increase in concentration. However, curcumin nanosuspension have shown significant $(\mathrm{P}<0.05)$ anti-bacterial activity against Escherichia coli at various concentrations in comparison with ethanolic solution of curcumin (Fig. 3).

Table 1 Anti-bacterial activity of anionic surfactant based topical curcumin nanosuspension and ethanolic solution of curcumin against Escherichia coli

\begin{tabular}{|l|l|l|l|}
\hline \multirow{2}{*}{ Samples } & \multicolumn{3}{|l|}{ Concentrations of samples } \\
\cline { 2 - 4 } & $50 \mu \mathrm{L}$ & $100 \mu \mathrm{L}$ & $150 \mu \mathrm{L}$ \\
\cline { 2 - 4 } & \multicolumn{3}{|l|}{ Mean inhibition zone diameters (in mm) } \\
\hline $\begin{array}{l}\text { Ethanolic solution of } \\
\text { curcumin (2 } \mathrm{mg} / \mathrm{ml})\end{array}$ & $14 \pm 0.20$ & $16 \pm 0.23$ & $16 \pm 0.19$ \\
\hline $\begin{array}{l}\text { Curcumin nanosuspension } \\
(2 \mathrm{mg} / \mathrm{ml})\end{array}$ & $21 \pm 0.12^{*}$ & $25 \pm 0.05^{*}$ & $29 \pm 0.08^{*}$ \\
\hline
\end{tabular}

$* \mathrm{P}<0.05$, as compared to ethanolic solution of curcumin.

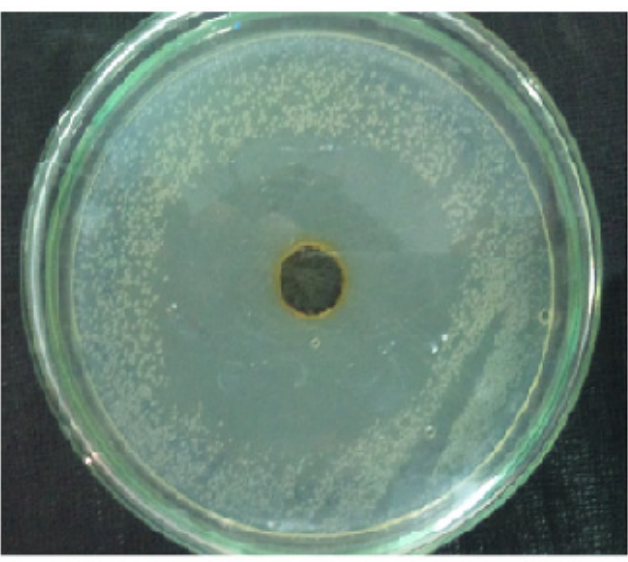

Ethanolic Solution of curcumin

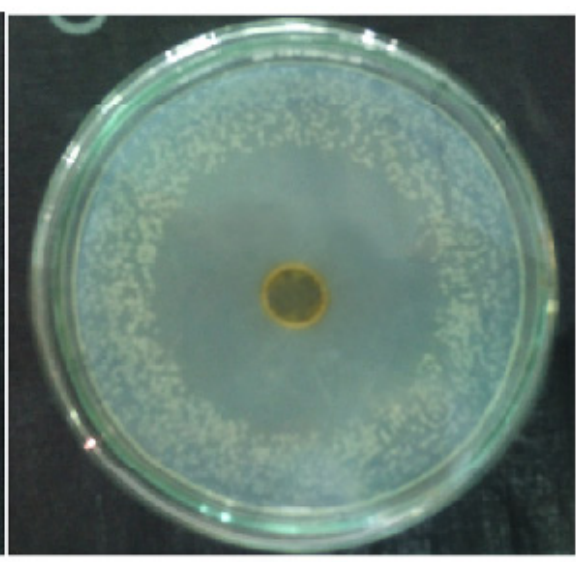

Curcumin Nanosuspension

Fig. 3 Anti-bacterial assay of curcumin nanosuspension against Escherichia coli in comparison with ethanolic solution of curcumin. 


\section{Conclusion}

We conclude that the prepared curcumin nanosuspension has produced average particle size less than $200 \mathrm{~nm}$ with narrow distribution range. Moreover, size reduction of curcumin in nano range have increased the surface area resulted in increased aqueous solubility and reactability of curcumin, which in turn have increased the potency of anionic surfactant based topical curcumin nanosuspension.

\section{References}

1 Aggarwal B.B., Sundaram C., Malani N., Ichikawa H., Curcumin: The Indian solid gold. Adv. Exp. Med. Biol. 2007; 595: 1-75.

2 Moorthi C., Kiran K., Manavalan R., Kathiresan, K., Preparation and characterization of curcumin-piperine dual drug loaded nanoparticles. Asian Pac. J. Trop. Biomed. 2012; 2: 841-848.

3 Moorthi C., Kiran K., Application of Plackett-Burman factorial design in the development of curcumin loaded Eudragit E 100 nanoparticles. Nano Biomed. Eng. 2013; 5: 28-33. http://nanobe.org

4 Zhou H., Beevers C.S., Huang S., Targets of curcumin. Curr. Drug Targets. 2011; 12: 332-347.

5 Moorthi C., Kathiresan K. Curcumin-Piperine / Curcumin-Silibinin/ Curcumin-Silibinin dual drug loaded nanoparticulate combination therapy: A novel approach to target and treat multidrug resistant cancers. J. Med. Hypotheses Ideas. 2013; 7: 15-20.

6 Moorthi C., Kathiresan K., Fabrication of highly stable sonication assisted curcumin nanocrystals by nanoprecipitation method, Drug Invention Today. 2013; http://dx.doi.org/10.1016/j.dit.2013.02.003.

7 Bhawana, Basniwal R.K., Buttar H.S., Jain V.K., Jain N., Curcumin nanoparticles: preparation, characterization, and antimicrobial study. J. Agric. Food Chem. 2011; 59: 2056-2061.

8 Mari selvam R., Ranjit Singh A.J.A., Kalirajan K., Anti-microbial activity of turmeric natural dye against different bacterial strains. Journal of Applied Pharmaceutical Science. 2012; 2: 210-212.

Copyright:(c) 2013 M. Chidambaram et al. This is an openaccess article distributed under the terms of the Creative Commons Attribution License, which permits unrestricted use, distribution, and reproduction in any medium, provided the original author and source are credited. 